\section{Lena Uller}

Unit of Respiratory Immunopharmacology, Dept Experimental Medical Science, Lund University, Lund, Sweden.

Correspondence: L. Uller, Unit of Respiratory Immunopharmacology, Dept Experimental Medical Science, BMC D12, Lund University, 22184 Lund, Sweden. E-mail: lena.uller@ med.lu.se

Statement of Interest: None declared.

\section{REFERENCES}

1 Lee CS, Yi EH, Lee JK, et al. Simvastatin suppresses RANTESmediated neutrophilia in polyinosinic-polycytidylic acid-induced pneumonia. Eur Respir J 2013; 41: 1147-1156.
2 Calvén J, Yudina Y, Hallgren $\mathrm{O}$, et al. Viral stimuli trigger exaggerated thymic stromal lymphopoietin expression by chronic obstructive pulmonary disease epithelium: role of endosomal TLR3 and cytosolic RIG-I-like helicases. J Innate Immun 2011; 4: 86-99.

3 Siednienko J, Halle A, Nagpal K, et al. TLR3-mediated IFN- $\beta$ gene induction is negatively regulated by the TLR adaptor MyD88 adaptor-like. Eur J Immunol 2010; 40: 3150-3160.

4 Brandelius A, Mahmutovic Persson I, Calvén J, et al. Selective inhibition by simvastatin of IRF3 phosphorylation and TSLP production in dsRNA-challenged bronchial epithelial cells from COPD donors. Br J Pharmacol 2013; 168: 363-374.

5 Ying S, O'Connor B, Ratoff J, et al. Expression and cellular provenance of thymic stromal lymphopoietin and chemokines in patients with severe asthma and chronic obstructive pulmonary disease. J Immunol 2008; 181: 2790-2798.

DOI: $10.1183 / 09031936.00133812$

\title{
Complexities of oestradiol pharmacology in pulmonary arterial hypertension
}

\section{To the Editor:}

We read with great interest the recent article by YUAN et al. [1]. The authors confirm previous observations that female sex and $\mathrm{E}_{2}$ (17 $\beta$-oestradiol) protect against a rat model of pulmonary hypertension $(\mathrm{PH})$, namely monocrotaline-induced PH (MCT$\mathrm{PH})$, and that the beneficial effects of $\mathrm{E}_{2}$ in MCT-PH are associated with increased lung nitric oxide, prostacyclin levels and reduced endothelin levels. Furthermore, based on $E_{2}$ plasma levels and altered expression of key enzymes, they suggest that $\mathrm{E}_{2}$ deficiency may play a role in MCT-PH.

We would like to draw attention to another dimension of $E_{2}$ pharmacology that adds to the complexity of $E_{2}$ in experimental $\mathrm{PH}$ and human pulmonary arterial hypertension (PAH), i.e. the metabolism of $E_{2}$ to biologically active metabolites. We also would like to discuss the concept that the overall effects of sex and $E_{2}$ in $\mathrm{PH}$ may depend on the model system.

Despite the fact that $E_{2}$ and female sex protect against MCT$\mathrm{PH}$, surprisingly $\mathrm{PAH}$ occurs more frequently in females than males. This incongruous finding is called the "PAH gender paradox" [2]. Even so, females with $\mathrm{PAH}$ have better right ventricular function and survival compared to males with PAH [3-5]. The apparent contradictions posed by the effects of female sex and oestrogens in experimental $\mathrm{PH}$ versus human $\mathrm{PAH}$ could be explained by the complexity of $\mathrm{E}_{2}$ metabolism, the opposing effects of $E_{2}$ versus its metabolites on endothelial vascular remodelling, limitations of the experimental models used, and the opposite roles that $\mathrm{E}_{2}$ may play in the pulmonary vasculature versus the right ventricle.

Several lines of evidence strongly suggest that the vascular protective effects of $\mathrm{E}_{2}$ are mediated largely by its downstream metabolites [2]. Notably, 2-methoxyestradiol (2ME, a major non-oestrogenic metabolite of $\mathrm{E}_{2}$ ) attenuates the development of and retards the progression of MCT- or hypoxia-induced $\mathrm{PH}$ in male rats and mediates the protective effects of $E_{2}$ in female rats with MCT- or bleomycin-induced PH [2]. Furthermore, $2 \mathrm{ME}$ is decisively more potent than $\mathrm{E}_{2}$ in increasing prostacyclin synthesis and release, in inhibiting endothelin synthesis in endothelial cells, in stimulating nitric oxide release from endothelium, and in inhibiting growth of systemic and pulmonary vascular smooth muscle cells [2]. Similar to the effects of $E_{2}$ in the YUAN et al. [1] study, inhibition of vascular remodelling by $2 \mathrm{ME}$ is also associated with down regulated expression of phosphorylated Akt and up regulated expression of cyclooxygenase-2 [6]. Thus, at least in part, the beneficial effects of oestradiol in MCT-PAH are mediated by $2 \mathrm{ME}$.

The hallmark vascular change in MCT-PH is medial thickening and neomuscularisation, with only mild endothelial damage that does not lead to occlusive proliferation of endothelial cells and formation of plexiform lesions. $\mathrm{E}_{2}$ and $2 \mathrm{ME}$ have opposing effects on endothelial cells: $E_{2}$ is promitogenic, proangiogenic and antiapoptotic, whereas $2 \mathrm{ME}$ is antimitogenic, antiangiogenic and proapoptotic. This may have significant ramifications for the overall effects of oestrogens in experimental $\mathrm{PH}$. We suggested that the effects of sex and oestrogens in experimental $\mathrm{PH}$ should be studied in a model of severe angio-proliferative PH (administration of vascular endothelial growth factor 2 receptor antagonist SU5416 and exposure to hypoxia) [2]. This model, despite its own limitations, more closely mimics the key vascular alterations and progressive and deadly character of disease seen in severe PAH in humans. In a recent study in this model (and contrary to the effects of sex and $E_{2}$ in the study by YUAN et al. [1]), we demonstrated that female rats develop more severe $\mathrm{PH}$ than males (numerous plexiform lesions in females compared to predominantly occlusive lesions in male rats), that in ovariectomised rats rescue treatment with $E_{2}$ exacerbates vascular lesions and 
$\mathrm{PH}$, and that $\mathrm{E}_{2}$ induces right ventricle-pulmonary vasculature uncoupling: despite more severe $\mathrm{PAH}$, females had significantly reduced right ventricular hypertrophy, and ovariectomy exacerbated the increase in right ventricular mass, an effect that was reversed by exogenous $\mathrm{E}_{2}[2,7]$. Therefore, MCT-PH may not be the best model for studying the complexities of oestrogen pharmacology in human PAH.

The concept that $\mathrm{E}_{2}$ deficiency is involved in MCT-PH may be correct; but this concept may be incorrect in human PAH. Monocrotaline is a toxin that, in male rats, reduces plasma testosterone levels by $50 \%$, and gonadal toxicity could be the reason for reduced $E_{2}$ levels. Furthermore, in the YUAN et al. [1] study, animals were not synchronised for oestrous cycle, and this may also have contributed to differences in plasma $E_{2}$ levels. It is noteworthy that recent clinical data suggest that female sex and increased, rather than decreased, $E_{2}$ levels and P450 Cyp19 (aromatase) activity are associated with greater risk of developing portopulmonary PAH [8].

Oestradiol may exert beneficial effects on the right ventricle, yet may exacerbate PAH and pulmonary vascular lesions. The question of whether oestradiol is good, bad, or both in human $\mathrm{PAH}$ requires further investigation. We view $\mathrm{E}_{2}$ as a doubleedged sword in human PAH.

\section{Stevan P. Tofovic* and Edwin K. Jackson ${ }^{\#}$}

*Dept of Medicine and Vascular Medicine Institute, Pittsburgh, PA, and "Dept of Pharmacology and Chemical Biology, University of Pittsburgh School of Medicine, Pittsburgh, PA, USA.

Correspondence: S.P. Tofovic, Division of Pulmonary Allergy and Critical Care Medicine, Dept of Medicine and Vascular Medicine Institute, 100 Technology Drive, Bridgeside 1, Room 542, Pittsburgh, PA 15129, USA. E-mail: tofovic@pitt.edu

Statement of Interest: Conflict of interest information can be found alongside the online version of this article at www.erj. ersjournals.com.

\section{REFERENCES}

1 Yuan $\mathrm{P}, \mathrm{Wu} \mathrm{WH}, \mathrm{Gao} \mathrm{L}$, et al. Oestradiol ameliorates monocrotaline pulmonary hypertension via NO, PGI2 and ET-1 pathways. Eur Respir J 2012; 41: 1116-1125.

2 Tofovic SP. Estrogens and development of pulmonary hypertension - interaction of estradiol metabolism and pulmonary vascular disease. J Cardiovasc Pharmacol 2010; 56: 696-708.

3 Benza RL, Miller DP, Gomberg-Maitland M, et al. Predicting survival in pulmonary arterial hypertension: insights from the Registry to Evaluate Early and Long-Term Pulmonary Arterial Hypertension Disease Management (REVEAL). Circulation 2010; 122: 164-172.

4 Humbert M, Sitbon O, Chaouat A, et al. Survival in patients with idiopathic, familial, and anorexigen-associated pulmonary arterial hypertension in the modern management era. Circulation 2010; 122: 156-163.

5 Escribano-Subias P, Blanco I, Lopez-Meseguer M, et al. Survival in pulmonary hypertension in Spain: insights from the Spanish registry. Eur Respir J 2012; 40: 596-603.
6 Barchiesi F, Jackson EK, Fingerle J, et al. 2-Methoxyestradiol, an estradiol metabolite, inhibits neointima formation and smooth muscle cell growth via double blockade of the cell cycle. Circ Res 2006; 99: 266-74.

7 Tofovic SP, Rafikova O, Jackson EK, et al. Estrogens exacerbates development of occlusive pulmonary arterial hypertension and formation of plexiform lesions. Am J Respir Crit Care Med 2012; 185: A6803.

8 Roberts KE, Fallon MB, Krowka MJ, et al. Pulmonary Vascular Complications of Liver Disease Study Group. Genetic risk factors for portopulmonary hypertension in patients with advanced liver disease. Am J Respir Crit Care Med 2009; 179: 835-842.

DOI: $10.1183 / 09031936.00164912$

\section{From the authors:}

We thank S.P. Tofovic and E.K. Jackson for their interest in our study [1]. It is well known that pulmonary arterial hypertension $(\mathrm{PAH})$ is a female-predominant disease [2-4], and this seems to indicate that oestrogens may be predisposing factors. However, this contrasts with the fact that females with $\mathrm{PAH}$ have preserved right ventricle function and survival compared with males with PAH [2-5], suggesting that oestrogens could be protective of PAH. So, oestrogens may be viewed as a double-edged sword in PAH. To explore the role of oestrogens in $\mathrm{PAH}$, we observed the effect of endogenous and exogenous $\mathrm{E}_{2}$ (17 $\beta$-oestradiol) in rats with monocrotaline (MCT)-induced pulmonary hypertension $(\mathrm{PH})$ [1]. Our results suggested similar protective roles of endogenous and exogenous $E_{2}$ in pulmonary vascular remodelling and right ventricular hypertrophy in MCT-induced PH rats, which is similar to the effect of $E_{2}$ reported in the study of UMAR et al. [6]. $E_{2}$ therapy has been shown to rescue both pulmonary vasculopathy and right ventricle function in rats with $\mathrm{PH}$. Thus, our results do not support the opinion expressed by S.P.Tofovic and E.K. Jackson that $E_{2}$ may have opposite roles in the pulmonary vasculature and the right ventricle.

In the study described by S.P. Tofovic and E.K. Jackson, 2methoxyoestradiol (2ME) attenuated the development of $\mathrm{PH}$ in rats and was decisively more potent than $\mathrm{E}_{2}$ in increasing prostacyclin and nitric oxide release, inhibiting endothelin-1 synthesis in endothelial cells, and inhibiting growth of systemic and pulmonary vascular smooth muscle cells. We do not exclude the possibility that the beneficial effects of oestradiol in MCT-induced $\mathrm{PH}$ are mediated, at least in part, by 2ME. Therefore, it is a good suggestion by S.P. Tofovic and E.K. Jackson that the effects of $E_{2}$ and its metabolites are studied in other animal models of $\mathrm{PH}$, which may further confirm the overall effects of $E_{2}$.

We have conducted a cohort study at our centre in which serum concentrations of oestradiol were measured in 120 adult fertile female patients with incident idiopathic PAH and 120 age- and sex-matched healthy female controls. Serum oestradiol concentrations were significantly decreased in $\mathrm{PAH}$ patients compared with controls (median (interquartile range): 184.6 (111.9-351.3) versus $\left.271.4(158.4-497.6) \mathrm{pmol} \cdot \mathrm{L}^{-1} ; \mathrm{p}<0.001\right)$. The same trend of was observed in serum oestradiol concentrations in 42 female patients with idiopathic PAH and 42 female controls studied in the follicular phase (median (interquartile range): 166.2 (114.1223.6) versus $\left.195.4(144.2-287.7) \mathrm{pmol} \cdot \mathrm{L}^{-1} ; \mathrm{p}=0.03\right)$ (fig. 1$)$. The data indicated that serum oestradiol concentrations were 